\title{
Unilaterales nävoides Teleangiektasie-Syndrom
}

\section{G. Wagner}

\section{Unilateral Nevoid Teleangiectasia Syndrome}

Zusammenfassung

Während ihrer ersten Schwangerschaft entwickelte sich bei der hier vorgestellten Patientin ein unilaterales nävoides Teleangiektasie-Syndrom. In der 29. SSW kam es zum intrauterinen Fruchttod, dessen Ursache nicht geklärt wurde. Mit einer Ausnahme fanden sich in der Literatur keine Berichte über kindliche Komplikationen bei schwangeren Frauen mit einem unilateralen nävoiden Teleangiektasie-Syndrom.
Abstract

During her first pregnancy the patient presented here developed a unilateral nevoid teleangiectasia syndrome. In the $29^{\text {th }}$ week of pregnancy intrauterine foetal death occurred. The cause of death was not determined. With a single exception, no reports were found in the literature of fetal complications in pregnant women with unilateral nevoid teleangiectasia syndrome.

\section{Einleitung}

Das unilaterale nävoide Teleangiektasie-Syndrom ist ein selten beschriebenes Krankheitsbild, das innerhalb der Gruppe der Teleangiektasien zu den primären oder idiopathischen Formen gezählt wird. Klinisch entwickeln sich streng einseitig, häufig im Bereich definierter Dermatome disseminiert stehende Teleangiektasien, die nebeneinander in Form zahlloser Einzelherde in punktförmigen, linearen oder verzweigten Strukturen angeordnet sein können. Gelegentlich finden sich in den betroffenen Arealen zusätzlich auch einzelne Naevi aranei. Dabei sind das Gesicht, der Hals sowie die Thoraxregion und die oberen Extremitäten am häufigsten betroffen [1]. Die Ätiopathogenese der Erkrankung ist nicht geklärt. Auffällig ist die gehäufte Manifestation in der Schwangerschaft und bei alkoholtoxischen Hepatopathien, so dass eine hormonelle, möglicherweise östrogenabhängige Auslösung diskutiert wird [2,3]. Das unilaterale nävoide Teleangiektasie-Syndrom ist eine Dermatose sui generis. Viszerale Manifestationen oder systemische Komplikationen gehören nicht zum typischen Krankheitsbild [1].

\section{Kasuistik}

\section{Anamnese}

In der 16. - 17. Woche ihrer ersten Schwangerschaft bemerkte die hier vorgestellte 31-jährige Patientin erstmals Hautveränderungen, die sich ausschließlich rechtsseitig am Arm und thorakal manifestiert hatten. Die Vorstellung in der Klinik erfolgte in der 25. SSW, wobei die Patientin zu diesem Zeitpunkt keine weitere Zunahme des Befundes mehr bemerkt haben wollte.

Die übrige Anamnese war vollständig unauffällig.

\section{Dermatologischer Befund}

Rechtsseitig in den Dermatomen $\mathrm{C}_{5-8}$ und $\mathrm{Th}_{1-3}$ fanden sich am Ober- und Unterarm einschließlich des Handrückens und der Fingerstreckseiten sowie am vorderen Dekolletee und über der Schulterblattregion disseminiert stehende oder auch auf umschriebenen Arealen zu größeren Gruppen zusammentretende Teleangiektasien. In die betroffenen Regionen vereinzelt eingestreut zeigten sich zusätzlich typische Naevi aranei (Abb.1,2).

Institutsangaben

Hautklinik, Zentralkrankenhaus Reinkenheide, Bremerhaven (Chefarzt: Dr. G. Wagner)

Widmung

Meinem verehrten Lehrer Prof. Dr. Theodor Nasemann zum 80. Geburtstag gewidmet.

Korrespondenzadresse

Dr. G. Wagner · Hautklinik · Zentralkrankenhaus Reinkenheide · Postbrookstraße $103 \cdot 27574$ Bremerhaven

Bibliografie

Akt Dermatol 2003; 29: 304-306 @ Georg Thieme Verlag Stuttgart · New York · ISSN 0340-2541 


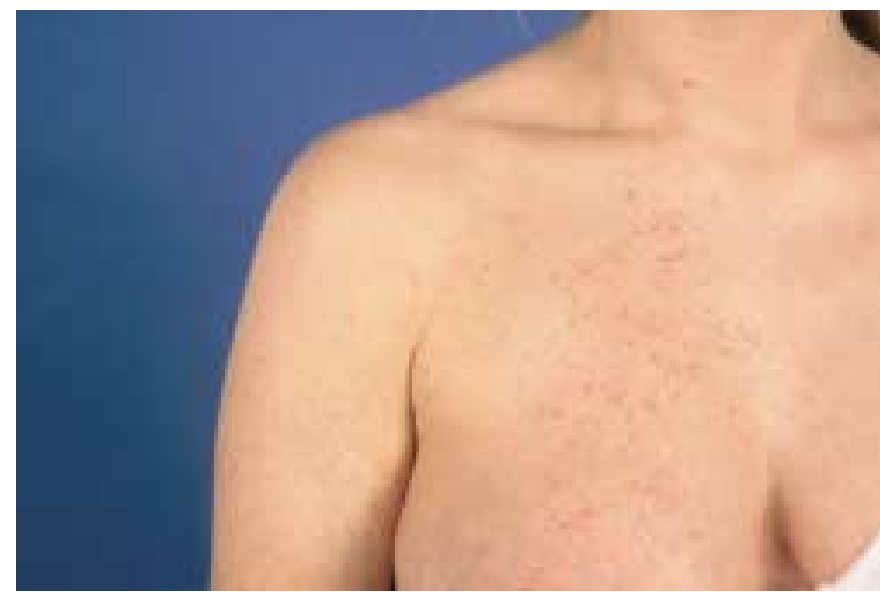

Abb. 1 Unilaterales nävoides Teleangiektasie-Syndrom.

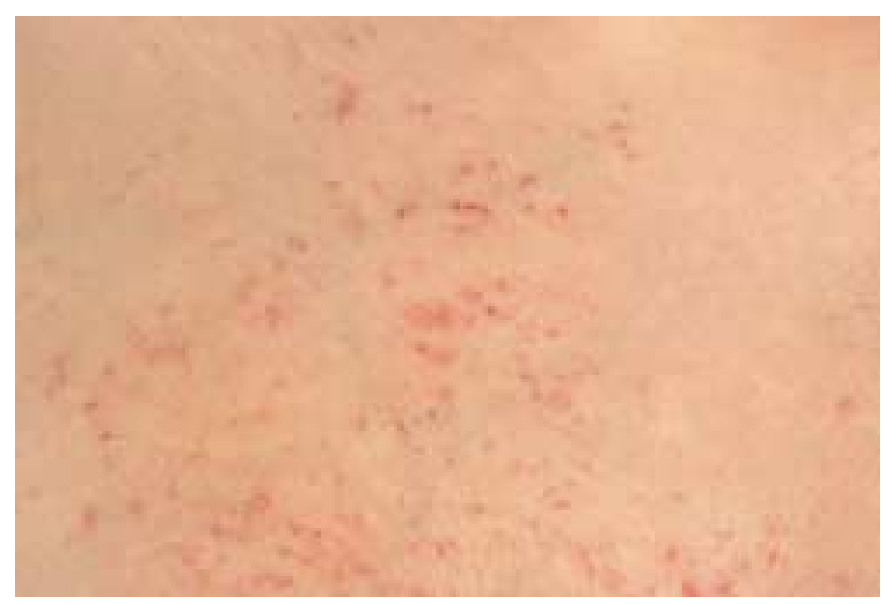

Abb. 2 Detailaufnahme vorderes Dekolletee.

\section{Histopathologischer Befund}

Bei unauffälliger Epidermis im oberen und mittleren Corium ektatische Gefäße. Vereinzelt umschrieben sehr diskretes, überwiegend lymphozytäres perivaskuläres Infiltrat.

\section{Laborbefunde}

Unauffälliges Blut- und Differenzialblutbild. Bilirubin und Lebertransaminasen ohne pathologische Befunde. Antinukleäre Antikörper nicht nachweisbar.

\section{Verlauf}

In der 29. SSW kam es zum intrauterinen Fruchttod, dessen Ursache nicht abgeklärt wurde. Im Verlauf der nächsten 3 Monate entwickelten sich die Teleangiektasien fast vollständig zurück.

\section{Diskussion}

Das unilaterale nävoide Teleangiektasie-Syndrom ist eine selten beobachtete, wenig bekannte und doch unverwechselbare Dermatose. Innerhalb der von Raff und Bardach zusammengestellten klinischen Kriterien ist die streng unilaterale Anordnung der Teleangiektasien für die Diagnose entscheidend. Typischerweise erkranken junge Erwachsene, seltener hingegen Kinder und Ju- gendliche. Neben zeitlich begrenzten oder chronisch-rezidivierenden Exazerbationen wurden auch spontane Rückbildungen der Teleangiektasien beobachtet. Viszerale Manifestationen gehören nach den Kriterien von Raff und Bardach nicht zum typischen Bild der Erkrankung [1].

Die Ätiologie und die Pathogenese des unilateralen nävoiden Teleangiektasie-Syndroms konnten bisher nicht geklärt werden. Das gehäufte Auftreten bei Frauen in der Schwangerschaft, die postpartale Rückbildung der Teleangiektasien und Rezidive bei erneuten Schwangerschaften sowie die Manifestation bei zumeist alkoholtoxischen Hepatopathien lassen an eine hormonabhängige Auslösung denken, wobei insbesondere den Östrogenen eine pathogenetische Bedeutung zugeschrieben wird [2-5]. Auch das vereinzelte Auftreten der Erkrankung bei Jugendlichen in der Pubertät unterstreicht die mögliche Bedeutung der Sexualhormone [6]. Die Untersuchungen von Uhlin et al. unterstützen ebenfalls die Theorie der endokrinologischen Induktion der Teleangiektasien. So konnten die Autoren eine deutliche Vermehrung der Östrogen- und Progesteronrezeptoren in den betroffenen Hautarealen bei unauffälliger Verteilung der Rezeptoren in normaler Haut nachweisen [2]. Allerdings konnten andere Autoren diese Befunde bei ihren Untersuchungen nicht nachvollziehen $[7,8]$. Unbekannt ist ferner der pathogenetische Mechanismus, der erklären würde, auf welche Weise Sexualhormone die Dilatation der Gefäße verursachen. Möglich erscheint dabei eine von Wilkin postulierte genetisch bedingte Verteilungsstörung Östrogen-empfindlicher Target-Zellen, die bei ansteigendem Östrogenspiegel für die Ausbildung der Teleangiektasien verantwortlich sein könnten [3]. Raff und Bardach halten darüber hinaus auch eine Dysfunktion vegetativer Nerven für möglich, da sie bei histologischen Untersuchungen weder cholinerge noch adrenerge Nervenfasern in den betroffenen Arealen eines Patienten nachweisen konnten. Des Weiteren diskutieren die Autoren die Bedeutung vasoaktiver Prostaglandine für die Pathogenese des unilateralen nävoiden Teleangiektasie-Syndroms [1].

Innerhalb der Gruppe möglicher Differenzialdiagnosen ist an den Morbus Osler, an den Naevus teleangiectaticus, an das Teleangiektasie-Ataxie-Syndrom sowie an essenzielle Teleangiektasien zu denken. Das unterschiedliche Manifestationsalter, die Verteilung der Morphen sowie das Auftreten assoziierter systemischer Erkrankungen erlauben im Allgemeinen jedoch eine sichere Abgrenzung der genannten Differenzialdiagnosen [6].

Nachdem es bei der hier vorgestellten Patientin zu einem intrauterinen Fruchttod gekommen war, wurde von Seiten der behandelnden Gynäkologen die Frage aufgeworfen, ob ein ursächlicher Zusammenhang zwischen diesem Ereignis und dem unilateralen nävoiden Teleangiektasie-Syndrom bestehen könnte. In der uns zugänglichen Literatur fand sich nur ein Bericht über eine Patientin, deren Kind unmittelbar nach der Geburt an den Folgen kardialer Fehlbildungen verstarb [9]. Da einerseits die möglichen Ursachen eines intrauterinen Fruchttodes außerordentlich vielfältig sein können und andererseits systemische Beteiligungen bei Patienten mit einem unilateralen nävoiden TeleangiektasieSyndrom nicht beschrieben wurden, muss letztendlich auch bei betroffenen schwangeren Frauen die Gefahr kindlicher Komplikationen verneint werden. 
Literatur

${ }^{1}$ Raff M, Bardach HG. Unilaterales nävoides Teleangiektasiesyndrom. Hautarzt 1982; 33: 148-151

${ }^{2}$ Uhlin SR, McCarthy KS. Unilateral nevoid teleangiectasia syndrome: The role of estrogen and progesterone receptors. Arch Dermatol 1983: 119: $226-228$

${ }^{3}$ Wilkin JK, Smith G, Cullison DA, Peters GE, Rodriquez-Rigau LJ, Feucht CL. Unilateral dermatomal superficial teleangiectasia. J Am Acad Dermatol 1983; 8: 468-477

${ }^{4}$ Labohm EB, Miseré J, Veen C. Unilaterales nävoides TeleangiektasieSyndrom. Hautarzt 1985; 36: 107-108

${ }^{5}$ Selmanoutse VJ. Unilateral nevoid teleangiectasia. Ann Intern Med 1970; 73: 87-90

\section{Preisausschreibung}

\section{Berliner Stiftung für Dermatologie}

Die Berliner Stiftung für Dermatologie, die im Dezember 1999 gegründet wurde und ihren Sitz an der Klinik und Poliklinik für Dermatologie am Universitätsklinikum Benjamin Franklin, Berlin, hat, vergibt ihren jährlichen Forschungspreis sowie Stipendien und unterstützt Forschungsprojekte, um die Wissenschaft auf dem Gebiet der Dermatologie und ihrer Grenzgebiete zu fördern.

\section{Aufgaben und finanzielle Mittel}

Gemäß ihrer Satzung hat die Stiftung als besonderes Ziel, finanzielle Ressourcen zu bündeln und die dermatologische Forschung, insbesondere auf dem Gebiet der Molekularbiologie und der Dermatopharmakologie, voranzutreiben. Ebenso soll die Umsetzung von neuen Erkenntnissen zur Diagnose und Behandlung von Hautkrankheiten in der Klinik gefördert werden.

Das Stiftungskapital beträgt derzeit ca. $650000 €$; davon steht

eine jährliche Förderungssumme von ca. $25000 €$ für zeitlich befristete Forschungsvorhaben klinischer und experimenteller Art, die jeweils durch das Kuratorium geprüft werden, zur Verfügung. Ein weiteres Ziel der Stiftung ist es, den internationalen Austausch von Wissenschaftlern auf dem Gebiet der dermatologischen Forschung zu fördern; dazu werden beispielsweise Stipendien für eine Weiterbildung in Deutschland über eine befristete Zeit vergeben.

Eine wichtige Aufgabe der Berliner Stiftung ist die Vergabe eines Stiftungspreises - mindestens alle 2 Jahre - in Höhe von $15000 €$ an jüngere Wissenschaftlerinnen und Wissenschaftler, die sich aufgrund persönlicher und wissenschaftlicher Leistungen in der Dermatologie hochrangig qualifiziert haben.

\section{Bewerbungen}

Bewerbungen um den Stiftungspreis und/oder um eine Unterstützung geeigneter wissenschaftlicher Projekte (Stipendien, Forschungsprogramme, Seminare) sind zu richten an das Kuratorium über seinen Geschäftsführer, Herrn Prof. Dr. Dr. C. C. Geilen, oder seinen Stellvertreter, Herrn Prof. Dr. C. E. Orfanos, beide in Berlin. Formlose Bewerbungen sind möglich, doch Anträge und weitere Informationen sind im Sekretariat oder über die Homepage der Stiftung zu erhalten, um den Vorgang zu vereinfachen (www.stiftung-dermatologie.de).

\section{Einsendeschluss für Bewerbungen ist der 31. August 2003.}

Bewerbungen um den jährlichen Stiftungspreis sollten möglichst das Curriculum und die neueste Publikationsliste des Bewerbers beigefügt werden.
${ }^{6}$ Abeck D, Thoms FH, Steinkraus V, Mensing H, Ring J. Unilaterales naevoides Teleangiektasie-Syndrom - Fallbeobachtung und Literaturübersicht. Z Hautkr 1991; 67: 688 - 690

${ }^{7}$ Hynes LR, Shenefelt PD. Unilateral nevoid teleangiectasia: occurrence in two patients with hepatitis C. J Am Acad Dermatol 1997; 36: $819-822$

8 Taskapan O, Harmanyeri Y, Sener O, Aksu A. Acquired unilateral nevoid teleangiectasia syndrome. Acta Derm Venerol (Stockh) 1997; 77: 62-63

${ }^{9}$ Lehner T, Tausch I, Audring H, Sönnichsen N. Naevoides Teleangiektatisches Syndrom (NTS)-assoziiert mit GraviditätDia-Klinik, X. Kongress der Gesellschaft für Dermatologie der DDR Dresden: 1990

\section{Mitteilung}

In einer akademischen Feierstunde hat am 29. Mai 2003 die Universität für Medizin und Pharmazie „Carol Davila“ in Bukarest (Rumänien) Herrn Prof. Dr. C. E. Orfanos, Direktor der Universitätshautklinik im Klinikum Benjamin Franklin der FU Berlin, die Ehrendoktorwürde verliehen. Die Zeremonie fand im Festsaal im Hauptgebäude der Universität unter der Leitung des Rektors, Professor L. M. Popescu MD, PhD statt, der auch die Laudatio hielt. In Anwesenheit des Dekans, mehrerer Prodekane und Mitglieder der Fakultät sowie vieler Studenten wurden die Verdienste des Laureaten gewürdigt und ihm die Urkunde überreicht. In seiner Antwort auf die Ehrung wies Prof. Orfanos auf die Bedeutung und den Vorbildcharakter eines wissenschaftlich orientierten akademischen Lebens für die heranwachsende junge Ärztegeneration in einem vereinigten Europa hin. In der Liste der insgesamt 27 „Doctores honoris causa“ in der Universität Bukarest sind unter anderem A. Butenandt (Deutschland) und Luc Montagnier (Frankreich) aufgeführt. 